\title{
Role of mineralocorticoid receptor on atrial structural remodeling and inducibility of atrial fibrillation in hypertensive rats
}

\author{
Shinpei Kimura, Masahiro Ito, Makoto Tomita, Makoto Hoyano, Hiroaki Obata, Limin Ding, \\ Masaomi Chinushi, Haruo Hanawa, Makoto Kodama and Yoshifusa Aizawa
}

Hypertension is well known to increase atrial fibrillation (AF) and the development of AF is associated with atrial chamber remodeling. Although mineralocorticoid receptor (MR) inhibition provides cardiovascular protection, the role of MR on atrial structural remodeling and inducibility of AF in hypertension remains unclear. Here, we investigated roles of the MR on atrial structural remodeling and inducibility of AF in hypertensive rats by using MR antagonist eplerenone (EPL). Dahl salt-sensitive (DS) rats were fed a normal-salt or a high-salt (HS) diet from 7 weeks, and a non-antihypertensive dose of EPL or vehicle was administrated from 13 weeks, at which time myocytes hypertrophy, interstitial fibrosis in the atrium and AF inducibility had increased, until 20 weeks. There was no significant difference in systolic blood pressure between DS+HS $(186 \pm 4 \mathrm{~mm} \mathrm{Hg})$ and DS+HS+EPL (184 $\pm 5 \mathrm{~mm} \mathrm{Hg})$ at 20 weeks. Burst atrial pacing demonstrated decreased AF inducibility in DS+HS+EPL (0 of 10) compared with DS+HS (7 of 10). Fibrosis and myocytes hypertrophy in the atrium were decreased in DS+HS+EPL with the reduction of atrial inflammatory cytokines. These beneficial effects of EPL were associated with less atrial oxidative stress, as assessed by 4-hydroxy-2-nonenal staining, and reduced activation of the Rho GTPase Rac1 in the atrium. Thus, MR has important roles in atrial structural remodeling and AF inducibility in Dahl rats. The effects of MR are associated, at least in part, with activation of Rac1-oxidative stress/inflammatory axis.

Hypertension Research (2011) 34, 584-591; doi:10.1038/hr.2010.277; published online 20 January 2011

Keywords: atrial fibrillation; atrial remodeling; mineralocorticoid receptor; Rac1

\section{INTRODUCTION}

Atrial fibrillation (AF) is the most common type of arrhythmia in clinical practice and is associated with increased risks for mortality and stroke. ${ }^{1-4}$ Hypertension is known as the most predominant disease to develop $\mathrm{AF}^{5,6}$ As the development of $\mathrm{AF}$ is associated with structural remodeling of the atrium, such as myocyte hypertrophy and interstitial fibrosis, preventing atrial remodeling may offer new therapeutic approaches to AF, the so called 'upstream therapy. ${ }^{7,8}$

Recent large clinical trials, both the Randomized Aldactone Evaluation Study (RALES) and Eplerenone Post-Acute Myocardial Infarction Heart Failure Efficacy and Survival (EPHESUS) studies, and other animal studies have demonstrated the beneficial effects of mineralocorticoid receptor (MR) antagonists on the prognosis and ventricular remodeling. ${ }^{9-12}$ However, little is known about the effects of the MR antagonist on atrial remodeling in hypertension. MR stimulation is linked to interstitial fibrosis, inflammation, hypertrophy and superoxide production in the heart ${ }^{13-15}$ all of which are known to provide a pathogenic substrate for $\mathrm{AF}^{8}{ }^{8}$ Thus, it is possible that MR is closely linked with atrial structural remodeling and AF inducibility, and MR inhibition might reverse them.

Growing evidence has revealed that reactive oxygen species (ROS) may have important roles in cardiovascular physiology and pathophysiology. ${ }^{16}$ ROS may act as signaling molecules for cardiac remodeling and/or injure myocardial structure directly. ${ }^{17,18}$ There are several sources of ROS in the heart, including mitochondrial electron transport system, nitric oxide synthase, xanthine oxidase and nicotinamide adenine dinucleotide phosphate (NADPH) oxidase. Racl, one of the small G proteins, has predominant roles in NADPH oxidase activity and is critical for generating ROS. ${ }^{19,20}$ Recently, some reports have revealed the relationship between MR activation and Rac1 activity. ${ }^{21,22}$ However, little is known about the relationship between MR and Rac1 in the heart.

In this study, we attempted to test the effect of MR inhibition on atrial structural remodeling and inducibility of AF in the low-aldosterone hypertension model, Dahl salt-sensitive (DS) rats. Furthermore, we evaluated the role of MR on ROS production and Rac1 activation in the atrium. 


\section{METHODS}

Animals

Six-week-old male DS rats were purchased from Japan SLC Company (Shizuoka, Japan) and were maintained in our animal facilities. Throughout the studies, all the animals were treated in accordance with the guidelines for animal experiments as laid out by our institute, and the Guide for the Care and Use of Laboratory Animals published by the US National Institutes of Health.

\section{Design of the study}

Experiment 1: atrial structural remodeling and AF inducibility in DS rats. DS rats were randomly divided into two groups: DS rats given an $8 \%$ high-salt (HS) diet (DS+HS, $n=5)$ and DS rats given a normal-salt (NS) $(0.3 \% \mathrm{NaCl})$ diet (DS+NS, $n=5)$ at 7 weeks. Systolic blood pressure was determined every week with a standard tail-cuff method. At 13 weeks, the studies of echocardiography and electrophysiology were performed followed by histological study for atrial structural remodeling.

Experiment 2: role of $M R$ on atrial structural remodeling and $A F$ inducibility in DS rats. DS rats fed the $8 \%$ HS diet from 7 weeks of age were randomized at 13 weeks to receive chow containing a non-antihypertensive dose of $200 \mathrm{mg}$ of eplerenone (EPL) (Pfizer Inc, Groton, CT, USA) per kg of body mass per day (DS+HS+EPL, $n=10$ ) or vehicle (DS+HS, $n=10$ ) until 20 weeks. The doses of EPL used in this study were determined on the basis of our preliminary experiments. Age-matched DS rats maintained with the normal salt diet served as the control (DS+NS, $n=7$ ). Systolic blood pressure was determined every week with a standard tail-cuff method. At 13 and 20 weeks, echocardiography was performed, and electrophysiological data and myocardial structural remodeling were subsequently evaluated.

\section{Blood pressure measurement and echocardiography}

Systolic blood pressure was measured every week with the use of a non-invasive tail-cuff system (BP-98A, Softron Co., Ltd, Tokyo, Japan). Echocardiography was performed as we have previously described. ${ }^{23}$ The left ventricular (LV) internal diameter in end diastole (LVDD) and end systole (LVSD), LV posterior wall thickness, LV fractional shortening (LVFS) and diameter of left atrium (LA) were calculated from M-mode echocardiograms in the LV long-axis view over three consecutive cardiac cycles.

\section{Electrophysiological study}

An electrophysiological study was performed as we have previously described. ${ }^{24}$ Baseline cardiac cycle intervals, including RR, PR and AV intervals, were measured in all rats. A wave duration was defined as the distance from the beginning of the A wave to the end of the A wave in the electrogram obtained from the right atrium. With burst pacing in the atrium at progressively shorter cycle lengths, the AV nodal Wenckebach point was determined. AF induction was defined as irregular activities, which were recorded via a pair of electrodes at the tip of catheter placed perpendicularly to that of stimulation. We repeated stimulation three times, at cycle lengths of $40 \mathrm{~ms}$ for 10,20 and $30 \mathrm{~s}$ of duration, and if $\mathrm{AF}$ was induced at least once, $\mathrm{AF}$ was considered to be inducible.

\section{Histopathological analysis}

The hearts were rinsed with saline. Both LA and RA were cut in cross-sections. Sections were fixed in $10 \%$ buffered formalin, embedded in paraffin and then stained with hemotoxylin-eosin and masson trichrome stain. Digital images of the stained sections were acquired with a microscope digital camera system (OLYMPUS BX60, OLYMPUS Corporation, Tokyo, Japan). Morphometrical analysis was carried out using MacSCORE software, version 2.5 (Mitani Corporation, Tokyo, Japan). The size of atrial myocytes and the fibrous tissue content in the atrium were analyzed as we have previously described. ${ }^{25}$

\section{Blood analysis}

Blood samples were collected from the inferior vena cava. The concentrations of aldosterone in plasma were measured with radioimmunoassay kits (SPAC-S Aldosterone kit, TFB, Tokyo, Japan). Plasma electrolytes, sodium and potassium, were measured using an electrolyte autoanalyzer (ATWill EA-06, Yokohama, Japan). Interleukin 6 and tumor necrosis factor (TNF)- $\alpha$ analysis was undertaken using the quantitative sandwich enzyme immunoassay technique (R \& D Systems, Minneapolis, MN, USA).

\section{Real time PCR analysis}

Total RNA was extracted from LA tissue and quantitative real time PCR analysis was performed using a Light Cycler-FastStart DNA MasterSYBR Green (Roche, Indianapolis, IN, USA). Primer sequences were as follows: TNF- $\alpha$, $5^{\prime}$-CTGTGCCTCAGCCTCTTCTCATTC-3' (sense) and $5^{\prime}$-TTGGGAACTTCTC CTCCTTGTTGG-3' (antisense); monocyte chemoattractant protein-1, 5'-CTGTCTCAGCCAGATGCAGTTAAT-3' (sense) and 5'-TATGGGTCAAGT TCACXATTCAAAG- $3^{\prime}$ (antisense); osteopontin, $5^{\prime}$-CGAGGTGATAGCTTGG CTTA-3' (sense) and 5'-ACTATCGATCGCATCCGACT-3' (antisense); angiotensin converting enzyme (ACE), 5-GCAGTACAAAGACTTGCCTG-3' (sense), $5^{\prime}$-TGGCAGAGGCTGACATGTTA- $3^{\prime}$ antisense). The absolute copy numbers of particular transcripts were calculated using Lightcycle software (Roche Applied Science, Indianapolis, IN, USA) with the standard curve approach.

\section{Immunohistochemistry}

Immunohistochemistry for 4-hydroxy-2-nonenal (HNE) was performed in both LA and LV. Briefly, non-specific binding was blocked with 10\% normal goat serum in phosphate-buffered saline ( $\mathrm{pH}$ 7.4) for $30 \mathrm{~min}$ before incubation with the monoclonal anti-HNE antibody (1:50 dilution, NOF Corporation, Tokyo, Japan) in phosphate-buffered saline overnight at $4^{\circ} \mathrm{C}$. Tissue sections were then incubated for $30 \mathrm{~min}$ at room temperature with an anti-mice immunoglobulin G secondary antibody using Histofine simple stain Rat MAX-PO (Nichirei, Tokyo, Japan). The sections were visualized under bright-field microscopy, and the images were recorded with the use of MacSCOPE (Mitani Corporation, Tokyo, Japan) after digitization of the images with a camera system. At least five fields were selected per rat and quantification was also performed blindly. Data are expressed as the percentage of the positive stained lesions.

\section{Rac1 activity}

Activated Racl was detected with a Racl Activation Assay Kit in both LA and LV (Upstate Biotechnology, Inc, Waltham, MA, USA) according to the manufacturer's instructions as we have previously described. ${ }^{26}$

\section{Statistical analyses}

Statistical analyses were performed using the unpaired student's $t$-test or one-way analysis of variance and the Bonferroni multiple comparison test. The differences were considered significant at $P<0.05$. All data are expressed as means \pm s.e.m.

\section{RESULTS}

Experiment 1: atrial structural remodeling and AF inducibility in DS rats

Blood pressure and cardiac morphology. General characteristics at 13 weeks are presented in Table 1. At 13 weeks of age, systolic blood pressure in the DS+HS group was significantly higher than that of the DS+NS group. The ratio of heart weight to body weight in the DS+HS group was significantly higher than that of the DS+NS group.

Echocardiography. The DS+HS group displayed cardiac hypertrophy with deteriorated systolic performance, as shown by echocardiography (Table 1). LA was larger in the DS+HS group than that of the DS+NS group at 13 weeks. Both LVDD and LVSD in the DS+HS group were significantly greater than those of the DS+NS group. Both IVS and posterior wall in the DS+HS group were significantly increased compared with those of the DS+NS group. LVFS in the DS+HS group was significantly lower than that of the DS+NS group (Table 1).

Atrial structural remodeling. Hematoxylin-eosin staining revealed that the size of the myocytes significantly increased in the DS+HS group compared with that of the DS+NS group (Figures 1a and b). Masson trichrome staining showed that the DS+HS group had significantly 
Table 1 Characteristics at 13 week

\begin{tabular}{|c|c|c|}
\hline & $D S+N S(\mathrm{n}=5)$ & $D S+H S(\mathrm{n}=5)$ \\
\hline \multicolumn{3}{|l|}{ Hemodynamics } \\
\hline BW (g) & $329 \pm 9$ & $321 \pm 11$ \\
\hline HW/BW (mg g $\left.{ }^{-1}\right)$ & $3.25 \pm 0.15$ & $4.27 \pm 0.26$ \\
\hline $\mathrm{SBP}(\mathrm{mm} \mathrm{Hg})$ & $146 \pm 14$ & $186 \pm 5^{*}$ \\
\hline \multicolumn{3}{|l|}{ Hematology } \\
\hline WBC $\left(10^{3} \mathrm{~mm}^{-3}\right)$ & $4880 \pm 548$ & $9800 \pm 1056^{* *}$ \\
\hline $\mathrm{RBC}\left(10^{4} \mathrm{~mm}^{-3}\right)$ & $784 \pm 121$ & $677 \pm 80$ \\
\hline $\mathrm{Hb}\left(\mathrm{g} \mathrm{ml}^{-1}\right)$ & $12.7 \pm 1.2$ & $11.8 \pm 0.5$ \\
\hline $\mathrm{Ht}(\%)$ & $49.8 \pm 1.9$ & $48.6 \pm 1.2$ \\
\hline PIt $\left(10^{4} \mathrm{~mm}^{-3}\right)$ & $71.2 \pm 10.5$ & $83.4 \pm 9.4$ \\
\hline \multicolumn{3}{|l|}{ Echocardiography } \\
\hline $\mathrm{LA}(\mathrm{mm})$ & $3.9 \pm 0.07$ & $4.2 \pm 0.05^{* *}$ \\
\hline LVDD (mm) & $6.4 \pm 0.14$ & $7.5 \pm 0.2^{* *}$ \\
\hline $\operatorname{LVSD}(\mathrm{mm})$ & $3.94 \pm 0.04$ & $5.30 \pm 0.16^{* *}$ \\
\hline LVFS (\%) & $38 \pm 0.01$ & $29 \pm 0.02 * *$ \\
\hline $\mathrm{PW}(\mathrm{mm})$ & $1.54 \pm 0.05$ & $1.90 \pm 0.05^{* *}$ \\
\hline \multicolumn{3}{|l|}{ Electrophysiology } \\
\hline $\mathrm{SCL}(\mathrm{ms})$ & $176.0 \pm 2.7$ & $179.0 \pm 2.7$ \\
\hline AV interval (ms) & $50.0 \pm 2.5$ & $56.0 \pm 4.8$ \\
\hline A wave duration (ms) & $31.0 \pm 2.7$ & $42.0 \pm 4.1^{*}$ \\
\hline WBP (bpm) & $526 \pm 70$ & $568 \pm 20$ \\
\hline $\mathrm{AF}$ & $0 / 5$ & $4 / 5^{*}$ \\
\hline
\end{tabular}

Abbreviations: AF, atrial fibrillation; AV, atrioventricular; BW, body weight; DS, Dahl salt; $\mathrm{HW}$, heart weight; HW/BW, ratio of heart weight to body weight; HS, high salt; Ht, hematocrit; LA, left atrial diameter; LVDD, left ventricular end diastolic diameter; LVSD, left ventricular end systolic diameter; NS, normal salt; PIt, platelet count; PW, diastolic posterior wall thickness; SBP, systolic blood pressure; SCL, sinus cycle length; WBP, AV nodal Wenckebach point. Data are mean $\pm \mathrm{s}$.e.

${ }^{*} P<0.05$ vs. DS+NS, ${ }^{*} P<0.01$ vs. DS+NS.

increased interstitial fibrosis in the atrial tissue compared with that of the DS+NS group (Figures 1c and d).

Electrophysiological evaluation. A wave duration in the DS+HS group was significantly longer than that of the DS+NS group (Table 1). AF was induced in four of five rats $(80 \%)$, which lasted for $24 \pm 6.0 \mathrm{~s}$ in the DS+HS group, but not in the DS+NS group (Table 1).

\section{Experiment 2: role of MR on atrial structural remodeling and AF inducibility in DS rats}

Blood pressure and cardiac morphology. General characteristics at 20 weeks are presented in Table 2. Systolic blood pressure in both the DS+HS group and DS+HS+EPL group were significantly higher than those of the DS+NS group throughout the experiment. There were no significant differences between the DS+HS group and the DS+HS+EPL group regarding systolic blood pressure at any age including at 20 weeks. The heart weight to body weight ratio in the DS+HS group was increased compared with that of the DS+NS group. EPL treatment tended to decrease this ratio in the DS+HS+EPL group, however there was no significant difference between them.

Echocardiography. Echocardiography revealed that LA, LVDD, LVSD and wall thickness were significantly increased, and LVFS was decreased in the DS+HS group compared with those of the DS+NS group at 20 weeks. EPL treatment in the DS+HS+EPL group significantly reduced the increases in LA, but not in LVDD, LVSD and wall thickness compared with those of the DS+HS group.

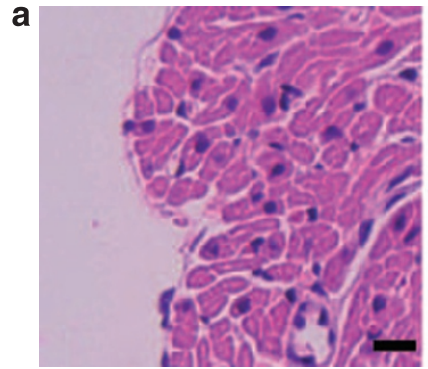

DS + NS

b

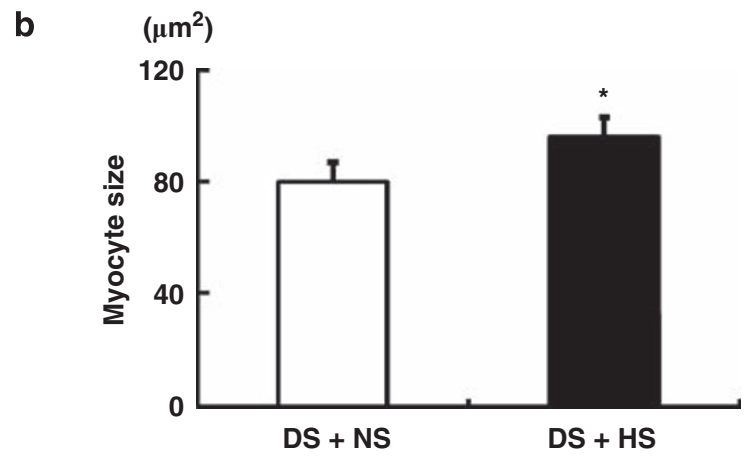

DS + NS

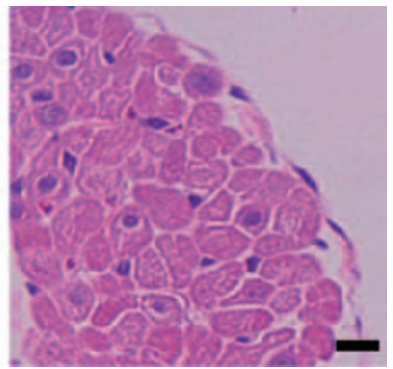

DS + HS

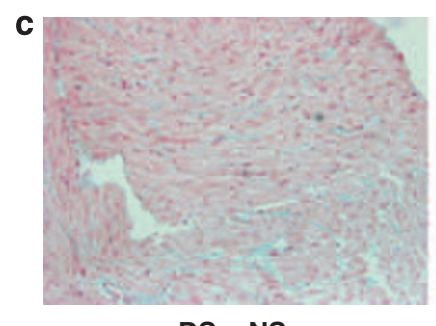

(\%)

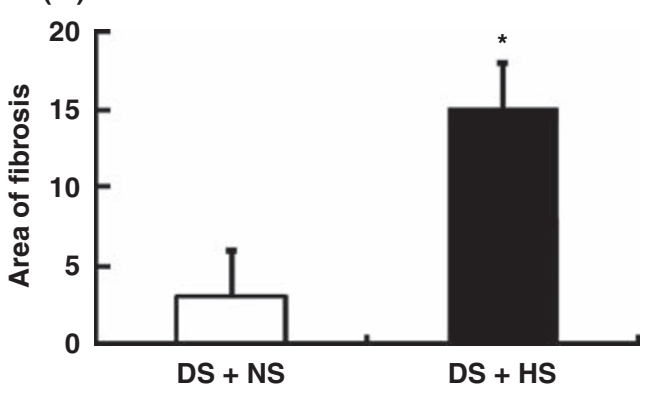

Figure 1 (a, b) Hematoxylin-eosin staining demonstrates that the size of myocytes increases in the DS+HS group at 13 weeks. ${ }^{*} P<0.05$ vs. DS+NS group. Bar $=0.01 \mathrm{~mm}$. (c, d) Masson trichrome staining demonstrates increased interstitial fibrosis in left atrial sections in the DS+HS group at 13 weeks. ${ }^{*} P<0.01$ vs. DS+NS group.

Myocyte size and myocardial fibrosis in the atrium. Hematoxylin-eosin staining revealed that the size of the atrial myocytes in the DS+HS group significantly increased compared with those of the DS+NS group and EPL treatment significantly attenuated this in the DS+HS+EPL group (Figures 2a and $\mathrm{b}$ ). Masson trichrome staining showed that interstitial fibrosis of the atrium in the DS+HS group had significantly increased compared with that of the DS+NS group and EPL treatment significantly decreased this in the DS+HS+EPL group (Figures $2 \mathrm{c}$ and d). 
Table 2 Characteristics at 20 week

$D S+N S(\mathrm{n}=7) \quad D S+H S(\mathrm{n}=10) \quad D S+H S+E P L(\mathrm{n}=10)$

\begin{tabular}{|c|c|c|c|}
\hline \multicolumn{4}{|l|}{ Hemodynamics } \\
\hline BW (g) & $388 \pm 15$ & $384 \pm 10$ & $390 \pm 10$ \\
\hline HW/BW (mgg $\left.{ }^{-1}\right)$ & $3.16 \pm 0.12$ & $4.79 \pm 0.22 * *$ & $4.46 \pm 0.16^{* *}$ \\
\hline $\mathrm{SBP}(\mathrm{mm} \mathrm{Hg})$ & $158 \pm 12$ & $186 \pm 4^{*}$ & $184 \pm 5^{*}$ \\
\hline \multicolumn{4}{|l|}{ Hematology } \\
\hline WBC $\left(10^{3} \mathrm{~mm}^{-3}\right)$ & $5200 \pm 689$ & $9250 \pm 578^{* *}$ & $6060 \pm 451 \ddagger$ \\
\hline $\mathrm{RBC}\left(10^{4} \mathrm{~mm}^{-3}\right)$ & $906 \pm 23$ & $723 \pm 57$ & $714 \pm 60$ \\
\hline $\mathrm{Hb}\left(\mathrm{g} \mathrm{ml}^{-1}\right)$ & 15. $7 \pm 0.4$ & $13.6 \pm 0.8$ & $13.7 \pm 0.9$ \\
\hline $\mathrm{Ht}(\%)$ & $48.7 \pm 1.0$ & $47.8 \pm 2.1$ & $45.4 \pm 2.1$ \\
\hline PIt $\left(10^{4} \mathrm{~mm}^{-3}\right)$ & $51.5 \pm 3.0$ & $83.9 \pm 6.1$ & $81.2 \pm 10.2$ \\
\hline \multicolumn{4}{|l|}{ Plasma analysis } \\
\hline $\mathrm{Na}$ (mequiv. $\mathrm{I}^{-1}$ ) & $147.0 \pm 1.8$ & $146.0 \pm 2.0$ & $146.2 \pm 2.2$ \\
\hline $\mathrm{K}$ (mequiv. $\mathrm{I}^{-1}$ ) & $4.2 \pm 0.3$ & $4.0 \pm 0.3$ & $4.0 \pm 0.3$ \\
\hline \multicolumn{4}{|l|}{ Echocardiography } \\
\hline $\mathrm{LA}(\mathrm{mm})$ & $4.0 \pm 0.08$ & $4.5 \pm 0.09 * *$ & $4.2 \pm 0.08^{\dagger}$ \\
\hline LVDD (mm) & $7.18 \pm 0.07$ & $7.89 \pm 0.07 *$ & $7.59 \pm 0.12$ \\
\hline LVSD (mm) & $4.34 \pm 0.13$ & $5.80 \pm 0.15^{* *}$ & $5.51 \pm 0.06$ ** \\
\hline LVFS (\%) & $39 \pm 1.7$ & $26 \pm 1.3^{* *}$ & $27 \pm 1.1^{* *}$ \\
\hline PW (mm) & $1.70 \pm 0.05$ & $2.0 \pm 0.03 * *$ & $1.9 \pm 0.03^{* *}$ \\
\hline \multicolumn{4}{|l|}{ Electrophysiology } \\
\hline $\mathrm{SCL}(\mathrm{ms})$ & $175.0 \pm 3.0$ & $177.0 \pm 1.6$ & $183.5 \pm 5.6$ \\
\hline AV interval (ms) & $51.0 \pm 2.0$ & $49.5 \pm 2.9$ & $45.5 \pm 1.2$ \\
\hline A wave duration (ms) & $30.0 \pm 1.7$ & $37.0 \pm 2.2^{*}$ & $31.5 \pm 1.1^{\dagger}$ \\
\hline WBP (bpm) & $612 \pm 37.6$ & $592 \pm 13.4$ & $566 \pm 22.6$ \\
\hline $\mathrm{AF}$ & $0 / 7$ & $7 / 10^{* *}$ & $0 / 10^{\ddagger}$ \\
\hline
\end{tabular}

Abbreviations: AF, atrial fibrillation; AV, atrioventricular; BW, body weight; DS, Dahl salt, $\mathrm{HS}$, high salt; $\mathrm{Ht}$, hematocrit; HW, heart weight; HW/BW, ratio of heart weight to body weight: LA, left atrial diameter; LVDD, left ventricular end diastolic diameter; LVSD, left ventricular end systolic diameter; NS, normal salt; PIt, platelet count; PW, diastolic posterior wall thickness; SBP, systolic blood pressure; SCL, sinus cycle length; WBP, AV nodal Wenckebach point.

Data are mean \pm s.e.

${ }^{*}<0.05$ vs. DS+NS, ${ }^{* *} P<0.01$ vs. DS+NS.

$P<0.05$ vs. DS+HS, $\neq P<0.01$ vs. DS+HS.

Electrophysiological evaluation. A wave duration in the DS+HS group was significantly longer than that of the DS+NS group, and EPL treatment significantly shortened it in the DS+HS+EPL group (Table 2). AF, which was not induced in the DS+NS group, was induced in 7 of 10 rats (70\%) in the DS+HS group and EPL treatment completely prevented this in the DS+HS+EPL group (0 of 10 rats, Table 2, Figure 3).

Blood analysis. The serum concentrations of aldosterone in both the DS+HS group and DS+HS+EPL group were significantly lower than that of the DS+NS group. EPL had no effect on the serum concentration of aldosterone (Figure 4). There was no significant difference in plasma electrolytes, or the serum concentration of TNF- $\alpha$ and interleukin-6 among the three groups (Table 2, Figure 4).

The mRNA expression and inflammation in the atrium. The levels of inflammation-related cytokine expressions in the atrium, including monocyte chemoattractant protein-1, osteopontin and TNF- $\alpha$ were significantly increased in the DS+HS group compared with those of the DS+NS group and EPL treatment significantly attenuated these (Figures $5 \mathrm{a}-\mathrm{c}$ ). The expression of ACE mRNA in the atrium was increased in the DS+HS group compared with that of the DS+NS group, and was significantly decreased by EPL treatment in the DS+HS+EPL group compared with the DS+HS group (Figure 5d). The amount of perivascular infiltration of inflammatory cells increased significantly in the DS+HS group compared with that of the DS+NS group, and EPL treatment in the DS+HS+EPL group markedly decreased this (data not shown).

Oxidative stress in the atrium. Staining for HNE in DS+HS group showed that the myocardium was stained diffusely in both LA and LV. The increased staining was markedly attenuated only in LA, but not in LV by EPL treatment (Figure 6a)

Racl activation in the atrium. Racl activation in LA was increased in DS+HS group, and this activation was inhibited only in LA, but not in LV by EPL in DS+HS+EPL group (Figures $6 \mathrm{~b}$ and c).

\section{DISCUSSION}

There have been accumulating studies investigating the role of MR in the heart. Previous papers have shown that MR stimulation with aldosterone and inappropriate salt intake caused cardiac hypertrophy and fibrosis, which were independent of blood pressure and preceded by perivascular and interstitial inflammation. ${ }^{13-15}$ These factors are known to have important roles in developing a pathogenic substrate for $\mathrm{AF}^{8}{ }^{8}$ Recently, Milliez et al. reported that spironolactone decreased atrial ectopy and fibrosis after myocardial infarction in rats. ${ }^{27}$ Shroff et al. revealed that EPL decreased AF maintenance and fibrosis in dogs with tachycardia-induced heart failure. ${ }^{28}$ However, the role of MR in the atrium, especially, in hypertension remains unclear. In this study, feeding a HS diet to DS rats from 7 to 13 weeks resulted in a significant increase in systolic blood pressure, myocyte hypertrophy and fibrosis in the atrium. Concomitant treatment with EPL from 13 to 20 weeks ameliorated myocyte hypertrophy and interstitial fibrosis in the atrium. These results indicated that MR activation is important in the etiology of atrial structural remodeling in DS rats. We also demonstrated that the MR antagonism markedly prevented $\mathrm{AF}$ inducibility in this model. Of note, inflammatory changes in the atrium were attenuated by MR inhibition with decreased expression of monocyte chemoattractant protein-1, osteopontin and TNF- $\alpha$. Our group previously demonstrated that EPL attenuated myocardial fibrosis and reduced LV mRNA expression of TGF- $\beta$ in rats in a postmyocarditis heart failure model. ${ }^{29}$ We evaluated the mRNA expression of TGF- $\beta$ in the heart, however the results were not uniform (data not shown). The number of WBCs in plasma decreased significantly in the EPL treated group compared with that of the non-treated group. The mechanism of this phenomenon was not revealed in our experiments. Peripheral blood mononuclear cells have been shown to release proinflammatory cytokines, which may have an important role in the process of LV remodeling. ${ }^{30}$ Although the fraction of WBC was not examined, there was no significant difference in systemic levels of proinflammatory cytokines in any of the groups. Thus, we believe that there was no significant relationship between the number of WBCs and the change of phenotypes in our experiments.

Recent studies indicate a mechanistic link between the inflammatory processes and the development of $\mathrm{AF}^{31,32}$ Frustaci et al. first demonstrated a high prevalence of inflammatory infiltrates, myocyte necrosis and fibrosis in atrial biopsies from patients with lone AF, but not in atrial biopsies from control patients. ${ }^{33}$ Other papers have reported that an increase in high-sensitivity C-reactive protein levels is an independent risk factor for future $\mathrm{AF}$ among patients with sinus rhythm. ${ }^{34,35}$ Whether inflammation is a consequence or a cause of AF is still controversial. However, our study demonstrated that inflammation-mediated atrial structural changes have important roles in DS 


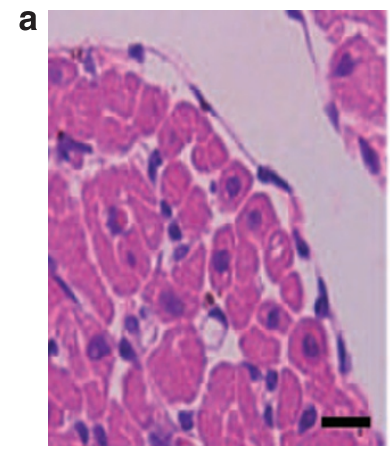

DS+NS

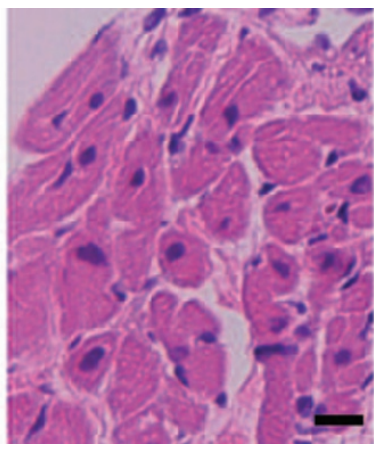

DS+HS

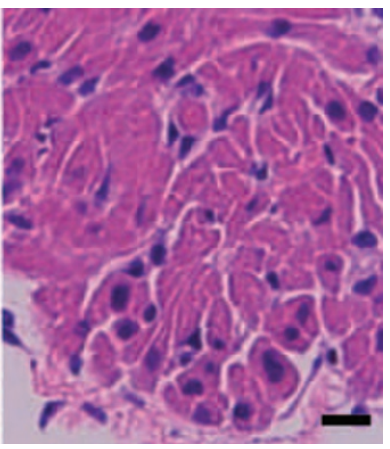

DS+HS+EPL

b $\left(\mu m^{2}\right)$

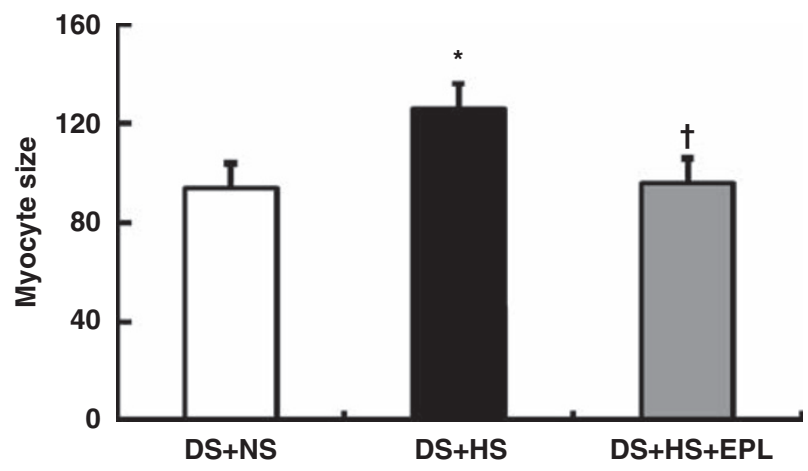

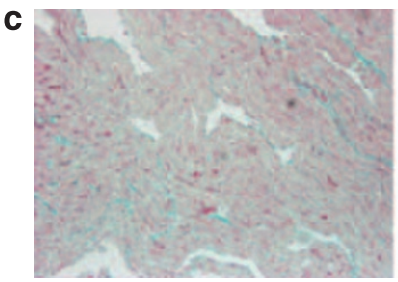

DS+NS

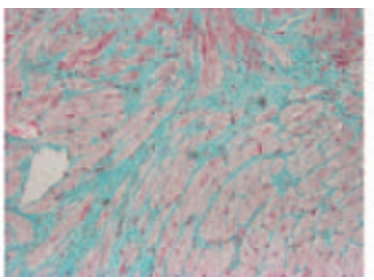

DS+HS

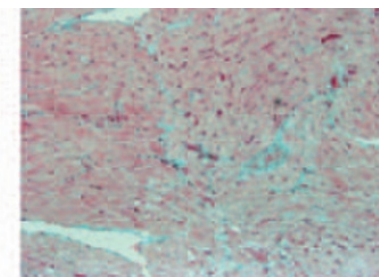

DS+HS+EPL

d (\%)

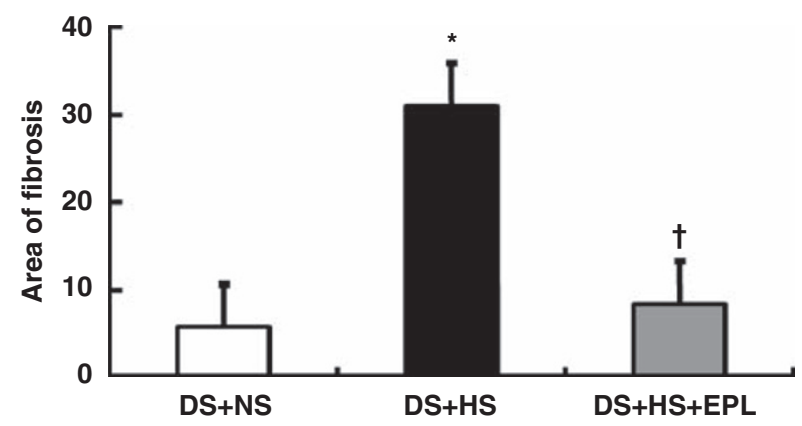

Figure 2 (a, b) Hematoxylin-eosin staining demonstrates that the size of myocytes increased in the DS+HS group, and EPL treatment markedly attenuates this in the DS+HS+EPL group at 20 weeks. ${ }^{*} P<0.05$ vs. DS+NS, ${ }^{\dagger} P<0.05$ vs. DS+HS. Bar $=0.01 \mathrm{~mm}$. (c, d) Masson trichrome staining demonstrates increased interstitial fibrosis in left atrial sections in the DS+HS group, and EPL treatment markedly attenuates this in the DS+HS+EPL group at 20 weeks. ${ }^{*} P<0.01$ vs. DS+NS group, ${ }^{\dagger} P<0.01$ vs. DS+HS.

rats and therapy towards decreasing inflammation, including MR inhibition, have great possibilities in treating patients with AF. Especially, our results demonstrated for the first time that MR inhibition had reverse remodeling effects on both the atrial structural remodeling and AF inducibility caused by hypertension.
Recent large clinical trials have demonstrated the clinical importance of the MR antagonist, however, precise mechanisms of its beneficial action have not been fully understood. ${ }^{9,10}$ Interestingly, serum concentrations of the aldosterone level of the patients were not elevated in either the RALES or EPHESUS studies. We confirmed 
that the serum concentration of aldosterone did not increase in the DS+HS group compared with that of the DS+NS group. Recent basic studies have demonstrated that MR activation is important in the etiology of cardiovascular and renal diseases not only in highaldosterone status but also in low-aldosterone hypertension, ${ }^{12,36}$ which are consistent with our results. How is MR activated in lowaldosterone hypertension? The underlying mechanism of this phenomenon remains unknown. In the present study, the expression of ACE mRNA in the atrium was increased, and MR inhibition with EPL significantly decreased this. A previous study indicated the existence

a

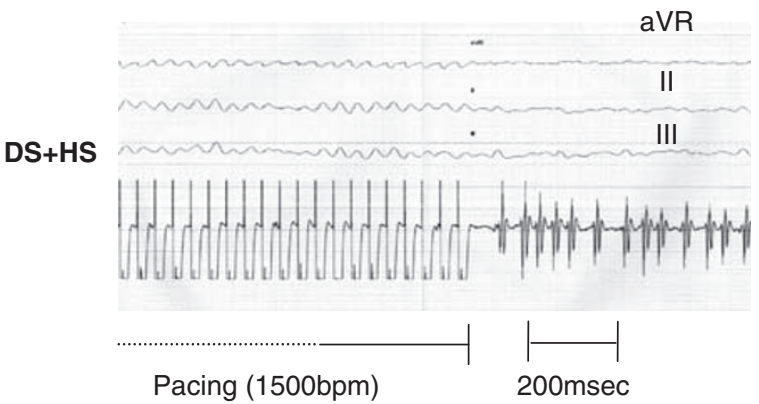

b

DS+HS+EPL

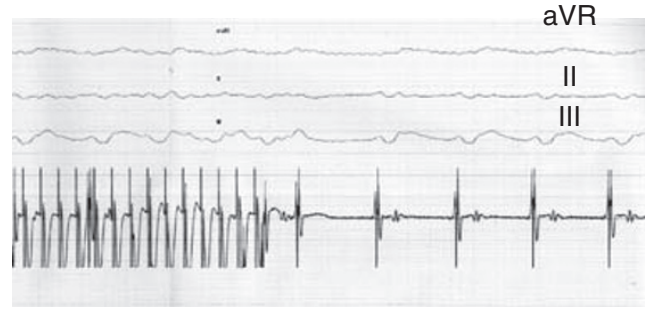

Pacing (1500bpm)

Figure 3 Stimulation for $\mathrm{AF}$ induction consisted of right atrial burst pacing at the cycle length of $40 \mathrm{~ms}$. (a) DS+HS group; (b) DS+HS+EPL group. AF is induced only in the DS+HS group. Paper speed, $200 \mathrm{~mm} \mathrm{~s}^{-1}$. of a positive feedback pathway from MR activation to ACE within the local cardiac renin-angiotensin-aldosterone system. ${ }^{37}$ These results suggested the existence and the importance of local tissue regulation of the rennin-angiotensin-aldosterone system in the atrium. Further study is needed to clarify the mechanism of MR activation in DS rats.

Another new finding of this study is that MR inhibition with EPL attenuated Racl activation in the atrium. This effect was associated with decreased myocardial oxidative stress, as assessed by HNE staining. Racl, which is one of the small G proteins, is essential for the assembly of the active NADPH oxidase complex, which may be a source of ROS. ${ }^{19,20}$ In addition to ROS production by NADPH oxidase, Rac1 also has been shown to participate in signaling pathways of LV hypertrophy and myocyte apoptosis. ${ }^{20,26}$ Recently, Adam et al. demonstrated that chronic cardiac overexpression of Racl in mice represented a novel model for AF. They also showed that left atrium of patients of AF exhibited up-regulation of Racl correlating with increased NADPH oxidase activity. ${ }^{38}$ In this study, increased atrial Rac1 activity in DS+HS group was significantly attenuated by EPL treatment with decreased staining for HNE. Thus, in the atrium, MR stimulation is associated with Racl activation, which has important roles in the atrial remodeling and inducibility of AF in DS rats through ROS production. Of note, it has also been demonstrated that aldosterone increased $A C E$ gene expression in cardiovascular tissue via Rac1 dependent mechanisms. ${ }^{22}$

Surprisingly, the beneficial effects of EPL on both atrial structural remodeling and the inducibility of AF were demonstrated in high blood pressure status. Of note, both increased ROS production assessed by HNE staining and activated Rac1 expression in DS+HS group were attenuated by EPL treatment only in LA, but not in LV. These results indicated that the beneficial effects of EPL were more marked in the atrium than in the ventricle under high blood pressure status, and that the regulatory mechanisms of atrial remodeling might be, at least in part, different from those of the ventricle.

In conclusion, MR inhibition with EPL reversed atrial structural remodeling and AF inducibility in DS rats, with a reduction of expression of inflammatory cytokines, ROS production and Rac1 activation in the atrium. Our findings suggest a new potential strategy targeting MR to treat patients with hypertension-mediated AF.

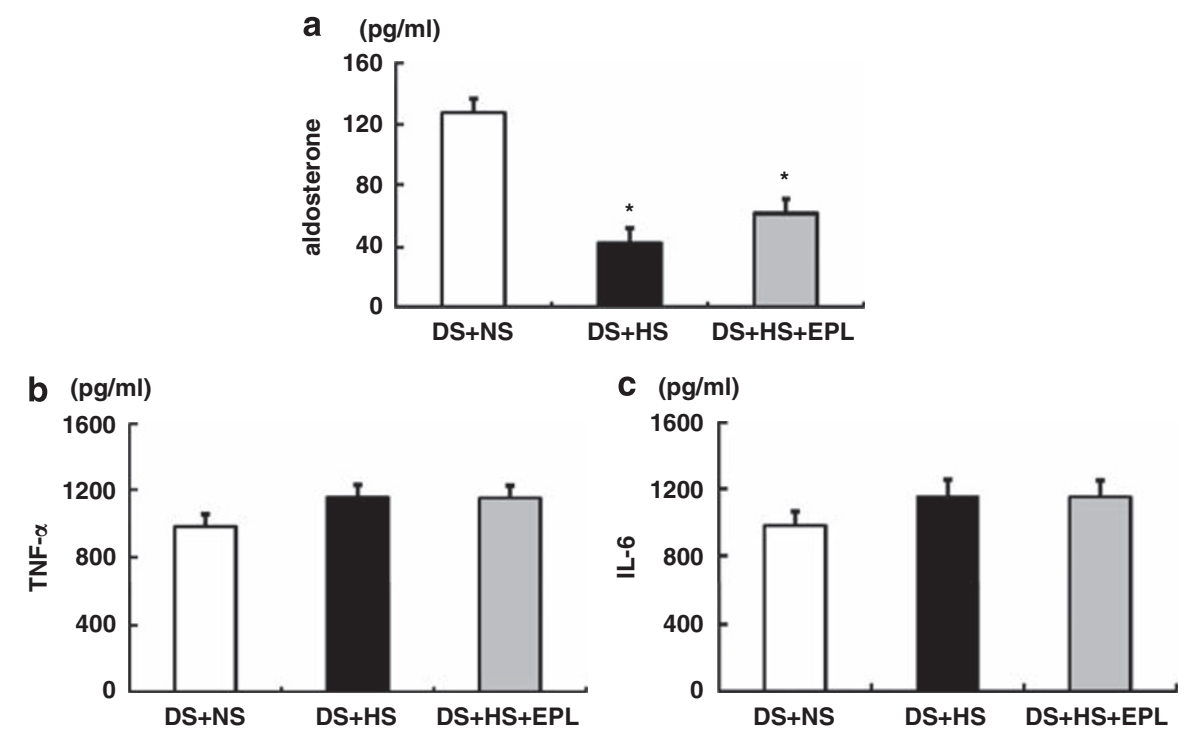

Figure 4 Aldosterone concentration (a), TNF- $\alpha$ concentration (b) and interleukin- 6 concentration (c) in plasma. ${ }^{*} P<0.05$ vs. DS+NS group. 

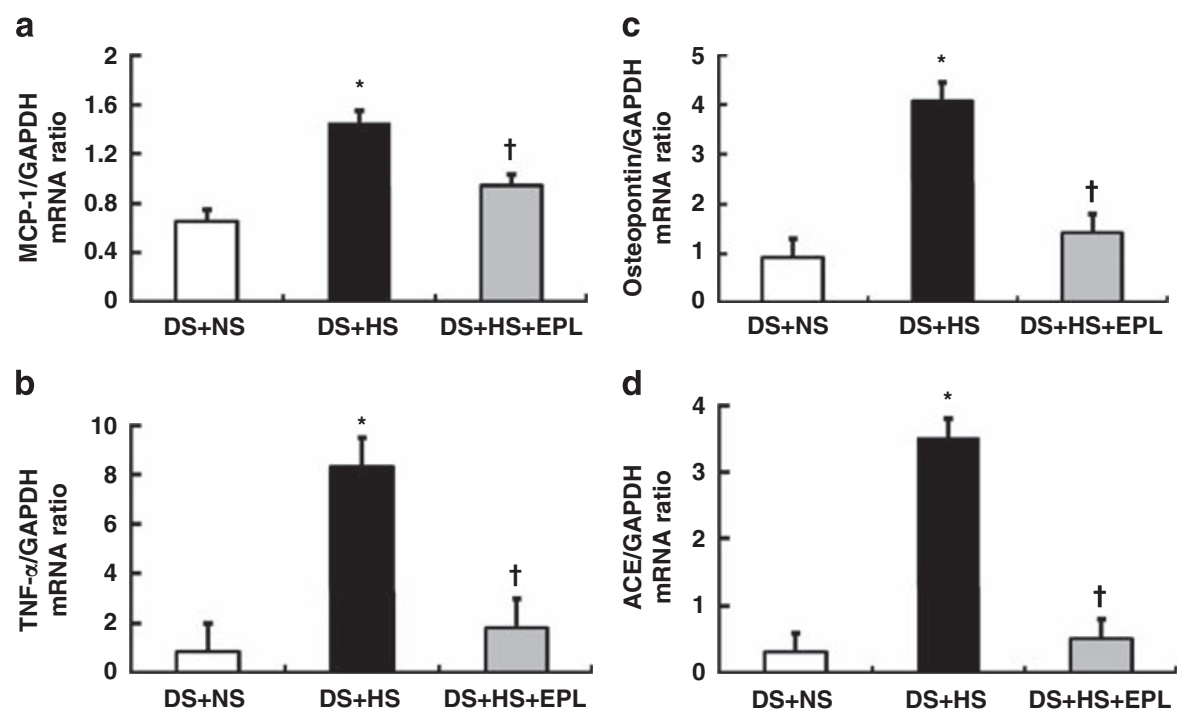

Figure 5 Quantitative analysis of the abundance of mRNAs for monocyte chemoattractant protein-1 (MCP-1) (a), TNF- $\alpha$ (b) osteopontin (c) and ACE (d) in the left atrium. ${ }^{*} P<0.01$ vs. DS+NS group, ${ }^{\dagger} P<0.01$ vs. DS+HS group.

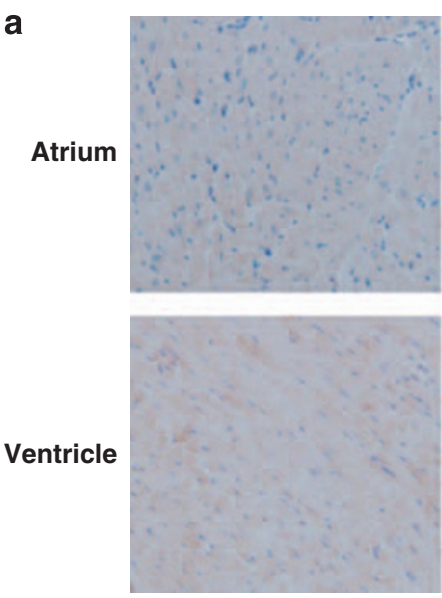

DS+NS
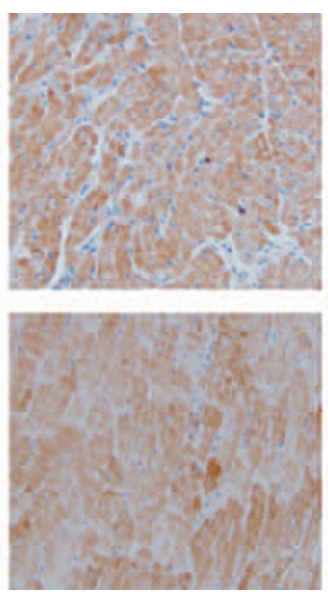

DS+HS
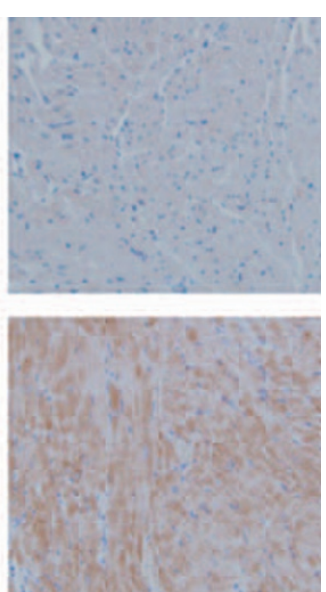

DS+HS+EPL

b Atrium

\section{Ventricle}

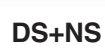

\section{DS+HS DS+HS+EPL}

C

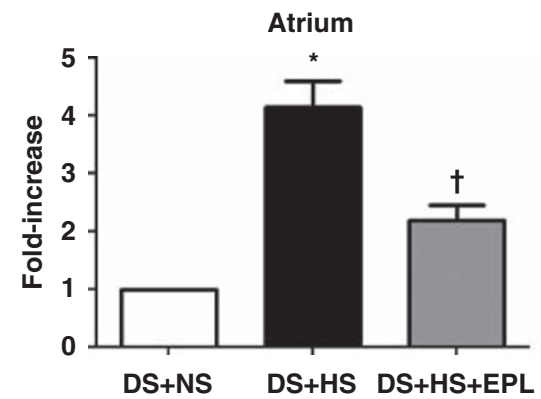

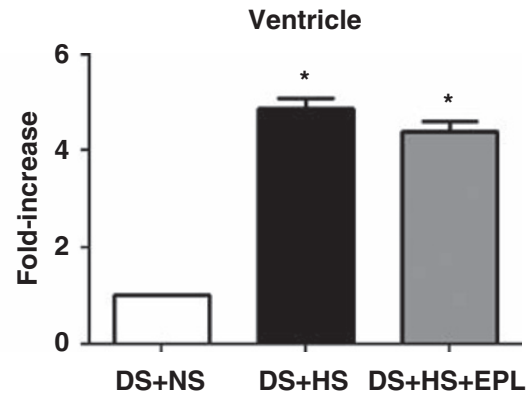

Figure 6 (a) Immunohistochemical staining for HNE in the atrium. Diffuse HNE staining is present in the atrium of DS+HS group, whereas staining is far less prominent in DS+HS+EPL group. HNE is localized predominantly to the myocardium. (b, c) Rac1 activity. Increased Rac1 activity in DS+HS group is inhibited by EPL treatment only in the atrium. ${ }^{*} P<0.05$ vs. DS+NS group, ${ }^{\dagger} P<0.05$ vs. DS+HS group. 


\section{CONFLICT OF INTEREST}

The authors declare no conflict of interest.

\section{ACKNOWLEDGEMENTS}

This work was supported in part by a grant from the Kanae Foundation for the Promotion of Medical Science and a grant for the Promotion of Niigata University Research Projects (to MI).

1 Lloyd-Jones DM, Wang TJ, Leip EP, Larson MG, Levy D, Vasan RS, D'Agostino RB, Massaro JM, Beiser A, Wolf PA, Benjamin EJ. Lifetime risk for development of atrial fibrillation: the Framingham Heart Study. Circulation 2004; 110: 1042-1046.

2 Benjamin EJ, Wolf PA, D'Agostino RB, Silbershatz H, Kannel WB, Levy D. Impact of atrial fibrillation on the risk of death: the Framingham Heart Study. Circulation 1998, 98: 946-952.

3 Stewart S, Hart CL, Hole DJ, McMurray JJ. A population-based study of the long-term risks associated with atrial fibrillation: 20-year follow-up of the Renfrew/Paisley study. Am J Med 2002; 113: 359-364.

4 Wolf PA, Abbott RD, Kannel WB. Atrial fibrillation as an independent risk factor for stroke: the Framingham study. Stroke 1991; 22: 983-988.

5 Go AS, Hylek EM, Phillips KA, Chang Y, Henault LE, Selby JV, Singer DE. of diagnosed atrial fibrillation in adults: national implications for rhythm management and stroke prevention: the Anticoagulation and Risk Factors in Atrial Fibrillation (ATRIA) Study. JAMA. 2001; 285: 2370-2375.

6 AFFIRM Investigators. Baseline characteristics of patients with atrial fibrillation: the AFFIRM Study. Am Heart J 2002; 143: 991-1001.

7 Healey JS, Baranchuk A, Crystal E, Morillo CA, Garfinkle M, Yusuf S, Connolly SJ. Prevention of atrial fibrillation with angiotensin-converting enzyme inhibitors and angiotensin receptor blockers: a meta-analysis. J Am Coll Cardiol 2005; 45: 1832-1839.

8 Nattel S, Burstein B, Dobrev D. Atrial remodeling and atrial fibrillation: mechanisms and implications. Circ Arrhythmia Electrophysiol 2008; 1: 62-73.

9 Pitt B, Zannad F, Remme WJ, Cody R, Castaigne A, Perez A, Palensky J, Wittes J. The effect of spironolactone on morbidity and mortality in patients with severe heart failure. Randomized Aldactone Evaluation Study Investigators. N Engl J Med 1999; 341: 709-717.

10 Pitt B, Remme W, Zannad F, Neaton J, Martinez F, Roniker B, Bittman R, Hurley S, Kleiman J, Gatlin M. Eplerenone, a selective aldosterone blocker, in patients with left ventricular dysfunction after myocardial infarction. N Engl J Med 2003; 348: 1309-1321.

11 Kuster GM, Kotlyar E, Rude MK, Siwik DA, Liao R, Colucci WS, Sam F. Mineralocorticoid receptor inhibition ameliorates the transition to myocardial failure and decreases oxidative stress and inflammation in mice with chronic pressure overload. Circulation 2005; 111: 420-427.

12 Nagata K, Obata K, Xu J, Ichihara S, Noda A, Kimata H, Kato T, Izawa H, Murohara T, Yokota M. Mineralocorticoid receptor antagonism attenuates cardiac hypertrophy and failure in low-aldosterone hypertensive rats. Hypertension 2006; 47: 656-664.

13 Brilla CG, Weber KT. Mineralocorticoid excess, dietary sodium, and myocardial fibrosis. J Lab Clin Med 1992; 120: 893-901.

14 Young M, Head G, Funder J. Determinants of cardiac fibrosis in experimental hypermineralocorticoid states. Am J Physiol 1995; 269: E657-E662.

15 Rocha R, Martin-Berger CL, Yang P, Scherrer R, Delyani J, McMahon E. Selective aldosterone blockade prevents angiotensin II/salt-induced vascular inflammation in the rat heart. Endocrinology 2002; 143: 4828-4836.

16 Sawyer DB, Siwik DA, Xiao L, Pimentel DR, Singh K, Colucci WS. Role of oxidative stress in myocardial hypertrophy and failure. J Mol Cell Cardiol 2002; 34: 379-388.

17 Pimentel DR, Amin JK, Xiao L, Miller T, Viereck J, Oliver-Krasinski J, Baliga R, Wang J, Siwik DA, Singh K, Pagano P, Colucci WS, Sawyer DB. Reactive oxygen species mediate amplitude-dependent hypertrophic and apoptotic responses to mechanical stretch in cardiac myocytes. Circ Res 2001; 89: 453-460.
18 Kwon SH, Pimentel DR, Remondino A, Sawyer DB, Colucci WS. $\mathrm{H}(2) \mathrm{O}(2)$ regulates cardiac myocyte phenotype via concentration-dependent activation of distinct kinase pathways. J Mol Cell Cardiol 2003; 35: 615-621.

19 Hordijk PL. Regulation of NADPH oxidases: the role of Rac proteins. Circ Res 2006; 98: 453-462.

20 Takemoto M, Node K, Nakagami H, Liao Y, Grimm M, Takemoto Y, Kitakaze M, Liao JK. Statins as antioxidant therapy for preventing cardiac myocyte hypertrophy. J Clin Invest 2001; 108: 1429-1437.

21 Shibata S, Nagase M, Yoshida S, Kawarazaki W, Kurihara H, Tanaka H, Miyoshi J, Takai Y, Fujita T. Modification of mineralocorticoid receptor function by Rac1 GTPase: implication in proteinuric kidney disease. Nat Med 2008; 14: 1370-1376

22 Iwashima F, Yoshimoto T, Minami I, Sakurada M, Hirono Y, Hirata Y. Aldosterone induces superoxide generation via Rac1 activation in endothelial cells. Endocrinology 2008; 149: 1009-1014

23 Mitsuma W, Ito M, Kodama M, Fuse K, Okamura K, Minagawa S, Kato K, Hanawa H, Toba K. Nakazawa M. Aizawa Y. Cardioprotective effects of recombinant human erythropoietin in rats with experimental autoimmune myocarditis. Biochem Biophys Res Commun 2006; 344: 987-994.

24 Hoyano M, Ito M, Kimura S, Tanaka K, Okamura K, Komura S, Mitsuma W, Hirono S, Chinushi M, Kodama M, Aizawa Y. Inducibility of atrial fibrillation depends not on inflammation but on atrial structural remodeling in rat experimental autoimmune myocarditis. Cardiovasc Pathol 2010; 19: e149-e157.

25 Okamura K, Ito M, Tanaka K, Chinushi M, Adachi T, Mitsuma W, Hirono S, Nakazawa M, Kodama M, Aizawa Y. effects of olmesartan and azelnidipine on atrial structural remodeling in spontaneously hypertensive rats. Pharmacology 2009; 83: 360-366.

26 Ito M, Adachi T, Pimentel DR, Ido Y, Colucci WS. Statins inhibit beta-adrenergic receptor-stimulated apoptosis in adult rat ventricular myocytes via a Rac1-dependent mechanism. Circulation 2004; 110: 412-418.

27 Milliez $P$, Deangelis N, Rucker-Martin C, Leenhardt A, Vicaut E, Robidel E, Beaufils $P$, Delcayre C, Hatem SN. Spironolactone reduces fibrosis of dilated atria during heart failure in rats with myocardial infarction. Eur Heart $J$ 2005; 26 : 2193-2199.

28 Shroff SC, Ryu K, Martovitz NL, Hoit BD, Stambler BS. Selective aldosterone blockade suppresses atrial tachyarrhythmias in heart failure. J Cardiovasc Electrophysiol 2006; 17: 534-541.

29 Wahed MI, Watanabe K, Ma M, Yamaguchi K, Takahashi T, Tachikawa H, Kodama M, Aizawa Y. Effects of eplerenone, a selective aldosterone blocker, on the progression of left ventricular dysfunction and remodeling in rats with dilated cardiomyopathy. Pharmacology 2005; 73: 81-88.

30 Mann DL. Inflammatory mediators and the failing heart: past, present, and the foreseeable future. Circ Res 2002; 91: 988-998.

31 Engelmann MD, Svendsen JH. Inflammation in the genesis and perpetuation of atrial fibrillation. Eur Heart J 2005; 26: 2083-2092.

32 Issac TT, Dokainish H, Lakkis NM. Role of inflammation in initiation and perpetuation of atrial fibrillation: a systematic review of the published data. J Am Coll Cardiol 2007 . 50: 2021-2028

33 Frustaci A, Chimenti C, Bellocci F, Morgante E, Russo MA, Maseri A. Histological substrate of atrial biopsies in patients with lone atrial fibrillation. Circulation 1997; 96 : 1180-1184.

34 Aviles RJ, Martin DO, Apperson-Hansen C, Houghtaling PL, Rautaharju P, Kronmal RA, Tracy RP, Van Wagoner DR, Psaty BM, Lauer MS, Chung MK. Inflammation as a risk factor for atrial fibrillation. Circulation 2003; 108: 3006-3010.

35 Asselbergs FW, van den Berg MP, Diercks GF, van Gilst WH, Van Veldhuisen DJ. C-reactive protein and microalbuminuria are associated with atrial fibrillation. Int J Cardiol 2005; 98: 73-77.

36 Nagase M, Shibata S, Yoshida S, Nagase T, Gotoda T, Fujita T. Podocyte injury underlies the glomerulopathy of Dahl salt-hypertensive rats and is reversed by aldosterone blocker. Hypertension 2006; 47: 1084-1093.

37 Harada E, Yoshimura M, Yasue H, Nakagawa O, Nakagawa M, Harada M, Mizuno Y, Nakayama M, Shimasaki Y, Ito T, Nakamura S, Kuwahara K, Saito Y, Nakao K, Ogawa H. Aldosterone induces angiotensin-converting-enzyme gene expression in cultured neonatal rat cardiocytes. Circulation 2001; 104: 137-139.

38 Adam 0, Frost G, Custodis F, Sussman MA, Schäfers HJ, Böhm M, Laufs U. Role of Rac1 GTPase activation in atrial fibrillation. J Am Coll Cardiol 2007; 50: 359-367. 